\title{
Impacto de los proveedores autorizados de la Universidad Nacional Autónoma de México en el desarrollo de colecciones de las bibliotecas de humanidades
}

\author{
Impact of the authorized providers of the Universidad Nacional Autónoma de México \\ in the collection development of the Humanities libraries
}

\section{María de los Ángeles Ciprés Oliva, Magdalena MiRAnda Díaz, Reyna Carolina GonzÁlez GarciA, Claudia del Carmen Perches GaLván, Norma Angélica QueZada RoJAS}

Biblioteca "Rubén Bonifaz Nuño", Instituto de Investigaciones Filológicas, Universidad Nacional Autónoma de México, México acipresoli@gmail.com

\begin{abstract}
Resumen
Se analiza el impacto de los proveedores autorizados por la Dirección General de Bibliotecas (DGB) de la Universidad Nacional Autónoma de México (UNAM) en el desarrollo de colecciones de las bibliotecas de su Subsistema de Humanidades. Se utilizó la observación directa y la aplicación de cuestionarios a los encargados del área de adquisiciones bibliográficas de las bibliotecas y a los proveedores autorizados. En general, el programa ha sido un éxito, pero los encargados de adquisiciones declaran que más de la mitad de la lista de proveedores no cubre el área geográfica de estudio de los proyectos de investigación que realiza su comunidad de usuarios. Es necesario, por tanto, asegurar que la lista de proveedores de editoriales relacionadas con las humanidades abarque en mayor medida a todos los países de América Latina. Se recomienda que la comisión de Biblioteca de cada biblioteca del Subsistema considere una reunión con los proveedores de material bibliográfico autorizados por la DGB para explicarles los proyectos y líneas de investigación de su dependencia y reforzar el conocimiento que tienen de la especialidad de cada biblioteca.
\end{abstract}

Palabras clave: Desarrollo de colecciones. Bibliotecas especializadas. Adquisiciones. Universidad $\mathrm{Na}-$ cional Autónoma de México. Méjico.

\section{Introducción}

La Universidad Nacional Autónoma de México (UNAM), es la institución de educativa más importante del país, en la que se llevan a cabo actividades de Investigación, Docencia y Difusión de la cultura.

Atiende cada semestre a estudiantes de bachiIlerato, licenciatura y posgrado; 30,000 docentes e investigadores, integran su planta académica, que en total conforma una comunidad de alrededor de 316,000 universitarios. En sus campus se imparten 71 licenciaturas, 9 carreras técni-

\begin{abstract}
The impact of the suppliers authorized by the Directorate General of libraries (DGB) of the National Autonomous University of Mexico (UNAM) in the development of collections of the libraries of the subsystem of Humanities at the University is analysed through direct observation and the application of questionnaires both to the acquisition staff and the suppliers authorized by the DGB. Acquisitions managers claim the program has been very successful, but also that more than half of the list of providers do not cover the geographic area of study of the research projects carried out by the community of users, and occasionally the have to seek their materials outside this list. Therefore, it is necessary to pay attention to the list of providers that work with editorials that publish in the field of Humanities, so that more countries in Latin America are covered. It is recommended that each subsystem library commission considers a meeting with the DGB-authorized bibliographic providers to explain them the research projects of their users and reinforce the knowledge they have of the specialty of each library.
\end{abstract}

Keywords: Collection development. Special libraries. Adquisitions. National Autonomous University of Mexico. Mexico.

cas, 149 maestrías, 72 doctorados y 48 programas de especialización. Los estudios profesionales se imparten en 22 escuelas y facultades distribuidas en Ciudad Universitaria y en el área metropolitana del Distrito Federal. Cuenta con 26 Institutos, 12 centros y siete programas de investigación en áreas científicas y humanísticas, que se encuentran vinculados con las diferentes Escuelas y Facultades.

Las actividades de investigación se agrupa en dos grandes subsistemas: Investigación Científica (SIC) e Investigación en Humanidades $(\mathrm{SIH})$, esta última regulada por la Coordinación 
de Humanidades que tiene entre sus objetivos principales fomentar el desarrollo académico y en particular la investigación en el área de las Humanidades y las Ciencias Sociales. Cabe mencionar que es en la UNAM en donde se lleva a cabo el $50 \%$ de la investigación del país.

En este contexto, las bibliotecas de los institutos del subsistema de humanidades apoyan de manera destacada a maestros e investigadores para que cuenten con los recursos y servicios de información que sustenten su formación académica y el desarrollo de su investigación.

El presente estudio de caso surgió como resultado del trato directo con los proveedores y editoriales autorizadas por la Dirección General de Bibliotecas (DGB) de la UNAM, para la compra de libros de la Biblioteca Rubén Bonifaz Nuño del Instituto de Investigaciones Filológicas de la misma universidad.

Aquí es importante hacer mención del papel que lleva a cabo la DGB dentro de la UNAM: es ella la encargada de coordinar el Sistema Bibliotecario de la Universidad con un total de 134 bibliotecas, conforme a las políticas generales que establece el Consejo del Sistema Bibliotecario, entre sus competencias se encuentra

Proporcionar asesorías sobre aspectos relacionados con edificios y automatización de bibliotecas, servicios bibliotecarios y de información, desarrollo de colecciones, organización de colecciones.

La DGB es también quien, con base en políticas internas, autoriza a los proveedores de material bibliográfico para que puedan ofrecer sus servicios a las diferentes bibliotecas que conforman el sistema bibliotecario de la UNAM.

De acuerdo al valor que tiene el papel de los proveedores para la adquisición del material bibliográfico, surge la inquietud de saber cómo logran conocer las líneas de investigación que se realizan en los institutos y centros, para poder ofrecer los materiales adecuados, considerando que son el sector al cual deben proporcionar un servicio, al estar registrados como autorizados por la DGB para la venta de libros.

Los responsables del área de adquisiciones de las bibliotecas saben que antes de comprar un libro debe ser revisado, valorado y aprobado por la Comisión de Biblioteca, o el coordinador del área académica solicitante. Sin embargo, esta dinámica no es suficiente, ya que año tras año se adquieren títulos retrospectivos que en su momento pasaron desapercibidos como un título de novedad, circunstancia que motivó a iniciar este trabajo con el objetivo de analizar el impacto sobre los proveedores autorizados por la DGB de la UNAM, y determinar su efecto y sus- tentabilidad para el desarrollo de colecciones, todo ello partiendo de la premisa que las labores más importantes del quehacer universitario son la investigación, la docencia, y la preservación y difusión del conocimiento y la cultura.

Para cumplir con este objetivo, el trabajo está estructurado en ocho apartados: introducción, metodología, bibliotecas del Subsistema de Humanidades, desarrollo de colecciones, resultados, discusión de resultados, conclusiones y recomendaciones.

\section{Metodología}

El estudio de caso es el principal objetivo hacia la valoración imparcial de los problemas operativos de las adquisiciones en el subsistema de humanidades. Su realización responde no solo a la vastedad y diversidad de proveedores sino principalmente a la necesidad de conocer si cubren las expectativas de las áreas responsables de las adquisiciones de las bibliotecas y el ejercicio de su presupuesto insoslayable en tiempo y forma para la elevación y calidad de los servicios ofrecidos.

La efectividad del estudio va en función de la metodología empleada, la cual está vinculada con los objetivos concretos que se persiguieron. En cuanto a ello es necesario puntualizar que no existen lineamientos metodológicos rígidos. (Izquierdo, 1995), "sino que estos pueden variar dependiendo de las peculiaridades del caso concreto". En el caso que nos ocupa, los lineamientos metodológicos que se describen a continuación se eligieron tomando en cuenta que nuestro objetivo central era recopilar datos de los servicios ofrecidos por parte de los proveedores y los responsables de adquisiciones a fin de detectar inconsistencias y lograr una mejora integral de la gestión de las adquisiciones y del desarrollo de colecciones en general.

Para la realización del estudio se utilizó un método directo de recolección de datos, el instrumento fue un cuestionario descripto-observacional.

\subsection{Instrumento}

El cuestionario, cuyo formato puede consultarse en el anexo, se diseñó de tal forma que permitiese obtener información directa de los encuestados. Se elaboró pensando en identificar aquellos aspectos que pudieran ayudar, por un lado, en la mejora substancial de los servicios ofrecidos; $y$, por otro lado, para ubicar las medidas que se pudieran tomar para mejorar la interacción con los proveedores y para abrir más espacios a otros nuevos que pudieran ofrecer mejo- 
res resultados. Las preguntas están redactadas y organizadas conforme a un orden y a unos objetivos predefinidos, lo cual facilitó la interpretación de los datos. Formalmente el instrumento constó de dos cuestionarios con reactivos de respuesta abierta.

\subsection{Aplicación}

Tras considerar la distancia a la que se encuentran algunas de las bibliotecas del Subsistema de Humanidades, dentro de la Ciudad de México con respecto al campus universitario, se decidió aplicarlo a las 16 bibliotecas localizadas en él. El cuestionario se aplicó a los encargados de adquisiciones bibliográficas de las bibliotecas del Subsistema, con el objetivo de conocer su punto de vista acerca del servicio y materiales que ofrecen (actuales, interesantes, etc.) a sus bibliotecas los proveedores autorizados.

Por otra parte, se aplicó también un cuestionario a 64 proveedores autorizados por la DGB, para conocer su labor y enterarnos de la situación de esta actividad. Con los resultados se pretendía identificar las variables clave, con la finalidad de estudiar los mecanismos de los servicios ofrecidos por los proveedores a las bibliotecas; y si éstas se encontraban satisfechas con los servicios obtenidos, o consideraban que hacían falta mejoras.

Es importante decir que, debido a que el cuestionario está formado por una cantidad pequeña de preguntas, el recuento y la codificación de las respuestas obtenidas se realizó de forma manual y sólo se requirieron medios automatizados para graficar y presentar los resultados.

La interpretación de resultados fue cuantitativa y cualitativa. La interpretación cualitativa consistió en el análisis de frecuencias de las respuestas obtenidas para cada reactivo (porcentajes sobre la muestra total). El análisis cualitativo se realizó, tal como señala Levin (1994) interpretando y comentando los resultados obtenidos.

\section{Bibliotecas del Subsistema de Humanidades}

Los Institutos y Centros de investigación que forman el Subsistema de Humanidades cuentan con bibliotecas especializadas que contribuyen de manera destacada, para que los profesionales de las humanidades cuenten con los recursos y servicios de información que apoyan la investigación y la formación académica, puesto que, como se mencionó anteriormente, cada instituto o centro está vinculado a las facultades de la universidad, en donde se forma a los futuros profesionales.
Para lograr este objetivo, entre las funciones que deben realizar, se encuentra el desarrollo de colecciones, actividad que lleva a cabo, en primera instancia, el coordinador de la biblioteca - el responsable del área de adquisiciones, junto con la comisión de biblioteca. Esta comisión integrada por el director del centro o instituto, el secretario académico, el secretario técni$\mathrm{co}$, el coordinador de la biblioteca, investigadores del centro o instituto, y dos miembros del personal académico y administrativo de la biblioteca.

En cada sesión de la comisión de biblioteca se levanta el acta correspondiente, en la que quedan asentados los detalles de la misma, así como los acuerdos de la sesión. Se pueden convocar reuniones extraordinarias de la comisión de biblioteca a petición del titular de la dependencia o por su representante en la comisión, o bien por un tercio de los integrantes de la misma, con objeto de discutir asuntos generales relacionados con la biblioteca y su desarrollo.

\section{Desarrollo de colecciones en las bibliotecas del Subsistema de Humanidades de la UNAM}

Como se mencionó anteriormente, el desarrollo de colecciones es una actividad en la que se involucran varias personas (coordinadores, responsables de adquisiciones, comisión de biblioteca). Su trabajo es seleccionar y evaluar cada novedad bibliográfica que se detecta en el mercado editorial y que esté relacionado con las necesidades de información, líneas de investigación que se estén desarrollando en el momento actual o que a futuro puedan ser temas de interés a investigar en el instituto o centro al cual pertenezca la biblioteca. Adicionalmente se encargan de señalar los temas que se deben fortalecer en el acervo bibliográfico existente.

Los responsables del área de adquisiciones están pendientes de detectar las novedades nacionales o extranjeras que los distribuidores y proveedores autorizados ofrecen para su adquisición. Igualmente, están alertas de las novedades que las universidades del país puedan otorgar en calidad de donación.

\section{Resultados}

Después de conocer algunos detalles del desarrollo de colecciones y de las bibliotecas del Subsistema de Humanidades de la UNAM, hablaremos del impacto de los proveedores autorizados por la Dirección General de Bibliotecas de esta Universidad en el desarrollo de colecciones de las bibliotecas de dicho Subsistema. 
El ochenta por ciento (80\%) de los encargados de adquisiciones de libros han invitado (influenciado) a proveedores externos para registrarse en la lista de proveedores autorizados. El objetivo de esta invitación por parte de los entrevistados es ampliar el abanico de posibilidades para la adquisición de material bibliográfico.

Sin embargo, de acuerdo a las respuestas de los proveedores autorizados por la DGB, el $62,71 \%$ no fueron invitados por ella a darse de alta como proveedores, frente al $37,28 \%$ que sí lo fue. Los proveedores se registran principalmente por iniciativa propia, considerando que la UNAM es una gran red de bibliotecas con una comunidad de usuarios multidisciplinaria que representa una gran posibilidad de negocio y un cliente muy importante.

Respecto al medio de comunicación que las bibliotecas utilizan para dar a conocer a los proveedores las líneas y proyectos de investigación de su comunidad de usuarios, el $80 \%$ de los encargados del área de adquisiciones de las bibliotecas del Subsistema lo hacen por medio de una explicación personal $(40 \%)$ o a través del correo electrónico (40\%). Sólo una pequeña parte lo hace remitiendo a la página web de la institución (20\%). Los encargados estiman que este método ha funcionado, ya que los proveedores han adquirido la experiencia que les permite entender los temas que son de interés para sus usuarios. También, a partir de las solicitudes de los investigadores, buscan al proveedor pertinente para surtir el material. Todos los proveedores, por su parte, declaran conocer las líneas de investigación —el 100\%.

Respecto al porcentaje de materiales a vistas y catálogos que se reciben afines a las necesidades de información de la comunidad de usuarios de las bibliotecas del Subsistema de Humanidades, el $80 \%$ de los proveedores ofrece obras relacionadas con las líneas y proyectos de investigación que realizan las comunidades académicas de cada biblioteca. El otro $20 \%$ de los proveedores entregan obras de carácter general o de otros temas que posiblemente pueden proponer para su compra en otras bibliotecas del mismo Subsistema.

Con respecto a esto, podemos decir que todos los proveedores -el 100\% - cuentan con personal con nivel de licenciatura que se encarga de seleccionar las obras a presentar en las bibliotecas de humanidades de la UNAM y en general para todas las bibliotecas de esta casa de estudios. Los proveedores consideran que el nivel intelectual de la persona que realiza esta actividad es importante, ya que es una tarea mental en la que se debe pensar la relación que existe entre la obra y el perfil de usuarios a quienes se quiere vender el material.

Además, todos los proveedores -el 100\%cuidan que las editoriales que ofrecen a las bibliotecas del Subsistema se apeguen al perfil de la comunidad de usuarios de dichas bibliotecas. Explican que, antes de ingresar una editorial en su catálogo, es examinada con base a su cartera de clientes.

Por otro lado, se reflejó que el $80 \%$ de los proveedores no alertan de las novedades que están por publicarse, y sólo el $20 \%$ lo hace. En opinión de los entrevistados esto no es un problema, dado que la comunidad académica se entera por sus propios medios y solicita la compra de las novedades conforme son publicadas en su lugar de origen. De acuerdo a nuestras observaciones hay investigadores que se suscriben a las alertas bibliográficas de las editoriales de su interés y así reciben la información de novedades; y con ella la biblioteca solicita el material.

Menos de la mitad de los proveedores -el $40 \%$ - están al día de las novedades que se publican -frente al $60 \%$ que no lo están. De acuerdo con la información proporcionada por los entrevistados, cuando los proveedores entregan el material a vistas, ofrecen libros ya publicados uno o dos años antes por las editoriales que ellos ofrecen. Posiblemente, esto se debe a que el proveedor no visitó la biblioteca a tiempo; o tal vez no registró que el material ya fue rechazado por la biblioteca en su momento y está tratando de colocar la venta. Por su parte, todos los proveedores -el 100\% - aseguran estar en permanente comunicación con las editoriales con las que trabajan, informan que realizan búsquedas bibliográficas en las bases de datos de las editoriales y consideran que están al día de las novedades y de las obras más importantes publicadas años antes.

Las editoriales que manejan los proveedores que cubren el área geográfica de interés de la comunidad de usuarios de las bibliotecas del Subsistema de Humanidades solo cubren el área geográfica de estudio de un $40 \%$ de las bibliotecas del Subsistema, mientras que el $60 \%$ no lo hace. De acuerdo con el criterio de los entrevistados es importante atender a las novedades que se publican en todos los países de América Latina. Con respecto a esto, observamos que la respuesta de los encargados se respalda con el material y catálogos que los proveedores presentan para su compra. Para satisfacer las necesidades de información de sus usuarios, los encargados buscan a los proveedores de acuerdo a su temática y cobertura, y les solicitan sus materiales, siendo generalmen- 
te atendidos. Al respecto, la mayor parte de los proveedores para la compra de libros declara trabajar con editoriales que cubren lugares de publicación a nivel mundial (el 40\%), mientras que el $39 \%$ lo hace con editoriales especializadas únicamente en Estados Unidos, Europa o América, y el $20,30 \%$ con otras que tienen una cobertura únicamente nacional.

En lo que se refiere a la frecuencia de compra de libros a proveedores no autorizados por la DGB en las bibliotecas del Subsistema de Humanidades, el $60 \%$ de las bibliotecas lo hacen de forma ocasional, y el $40 \%$ lo hace de forma frecuente. A juicio de los entrevistados, solo adquieren material de otros proveedores cuando se trata de editoriales que no son trabajadas por los autorizados o en el caso de que un investigador durante un viaje realice una compra directa. Con base en lo observado se puede decir que las editoriales que representan los proveedores no cubren todas las áreas de investigación de los usuarios de las bibliotecas del Subsistema. Los proveedores explican que venden material solo a algunas bibliotecas del Subsistema y, aunque han pensado ampliar su catálogo de editoriales, no han realizado su búsqueda pensando únicamente en el sector de humanidades, sino contemplando varias especialidades de la Universidad, ya que también tienen la posibilidad de ofrecer sus materiales a las preparatorias, colegios de ciencias y humanidades (nivel medio superior), y facultades de la UNAM.

\section{Discusión de los resultados}

En resumen, los encargados de adquisiciones declaran que más de la mitad de la lista de proveedores que los asisten no cubre el área geográfica de estudio de los proyectos de investigación que realiza la comunidad de usuarios. Por ello, es necesario ampliar la lista de proveedores que trabajan con las editoriales del campo de las humanidades para que abarquen en mayor medida a los países de América Latina: Cuba, Argentina, Colombia, Chile, Ecuador, Brasil, Belice, Costa Rica, El Salvador, Guatemala, Panamá, Nicaragua y Haití. Por su parte, la mayoría de los proveedores revelan que las editoriales con las que trabajan tienen cobertura mundial y una pequeña parte nacional, por lo que el déficit que exponen los encargados de adquisiciones no debería producirse. En cualquier caso, esta situación provoca que los encargados de adquisiciones ocasionalmente busquen su material bibliográfico fuera de la lista de proveedores.

Por último, aproximadamente la mitad de los proveedores registrados en el directorio autori- zado por la DGB trabajan con editoriales vinculadas con la temática de las bibliotecas del Subsistema de Humanidades. Sin embargo, llama la atención que los proveedores afirman atender solo algunas bibliotecas del Subsistema, circunstancia que contribuye a que los encargados de adquisiciones trabajen con todos los proveedores que les resulta posible.

\section{Conclusiones}

Los encargados de adquisiciones reciben asesoría de su comunidad académica y de la comisión de bibliotecas para realizar la selección de materiales, por lo que el proceso de selección se realiza entre los bibliotecarios, académicos y la comisión de bibliotecas.

Los responsables de adquisiciones han logrado mantener una buena comunicación con los proveedores, libreros y usuarios para la selección y adquisición de material, consiguiendo que el desarrollo de sus colecciones tome una forma física y tangible. La DGB de la UNAM ha evaluado el ingreso de cada proveedor que registra como autorizado para la venta de libros, logrando aumentar las editoriales, lugares de publicación y temática, reforzando las alternativas para la adquisición de los libros, no solo de las bibliotecas del Subsistema de Humanidades sino de toda la red de bibliotecas de la UNAM.

También es evidente que los encargados de adquisiciones mantienen una comunicación constante con los proveedores para transmitirles las necesidades de información de su comunidad de usuarios, consiguiendo que las conozcan y presenten las obras más apropiadas para su adquisición. Sin embargo, resulta notorio que los proveedores no alertan de las novedades que están por publicarse y no alcanzan a presentar las novedades que se publican en las editoriales que trabajan. Además, los responsables de adquisiciones manifestaron que, dentro de las editoriales que manejan los proveedores autorizados que los visitan, más de la mitad de la lista no cubre los países de América Latina. Esta situación causa que ocasionalmente busquen su material bibliográfico fuera de la lista de proveedores.

Este panorama nos permite decir que la Dirección General de Bibliotecas de la UNAM ha cumplido, y ha logrado fortalecer la Red de Bibliotecas de la Universidad, al ofrecer un catálogo amplio de proveedores de material bibliográfico autorizado. De igual manera, los encargados de adquisiciones de cada biblioteca del Subsistema se esmeran y esfuerzan por cubrir las carencias referentes a los proveedores sin alejarse de lo dispuesto por la DGB, respon- 
diendo a las necesidades de información de sus usuarios en tiempo y forma, con base a nuestra observación, y auxiliándose ocasionalmente de la compra directa por parte de los investigadores durante sus viajes.

\section{Recomendaciones}

A partir de las conclusiones de la investigación, se recomienda que la Comisión de Biblioteca de cada Instituto del Subsistema considere una reunión con los proveedores de material bibliográfico autorizados para explicarles los proyectos y líneas de investigación de su dependencia. Esto reforzaría el conocimiento que tienen de la especialidad de cada biblioteca; y permitirá que los proveedores identifiquen con exactitud los países de estudio de cada entidad.

También sería conveniente realizar un estudio de las necesidades de información de la comunidad de usuarios de cada biblioteca, para implementar un plan de desarrollo de colecciones que responda al usuario y sus necesidades específicas. Este plan podrá ser ejecutado por el personal involucrado en este proceso. En este sentido, se aconseja también realizar un estudio del perfil de los proveedores para aumentar los países de publicación de las obras que ofrecen, así como cubrir de forma más completa las áreas de investigación de la comunidad académica de cada dependencia del Subsistema de Humanidades.

Finalmente, convendría realizar una evaluación de colecciones para implementar un plan de adquisiciones con conocimiento de la colección existente, y de las fortalezas y debilidades existentes en el proceso de selección.

\section{Referencias}

Busha, Charles (1990). Métodos de investigación en bibliotecología: técnicas e interpretación. México: UNAM, 1990.

Fuentes, J.J. (2007). Las definiciones de la colección de materiales de la biblioteca: los conceptos de desarrollo y gestión de las colecciones: un estado de la cuestión. /I Boletín de la Asociación Andaluza de Bibliotecarios. 22:88-89, 89-108.

Gavilán, César Martín (2008). Selección y adquisición de materiales criterios para la formación, mantenimiento y evaluación de la colección bibliográfica. http://eprints.rcli s.org/14882/1/selcyadq.pdf (2014-01-18).

Institutos de Humanidades. http://www.dgbiblio.unam.mx/in dex.php/bibliotecasunam (2014-01- 25)

Kanwal, A. (2005). Developments in the philosophy of collection management. // Collection Building. 24:4 (2005) 112-116.

Negrete, María del Carmen (2003). El desarrollo de colecciones y la selección de recursos en la biblioteca universitaria. México: Centro Universitario de Investigaciones Bibliotecológicas, 2003.
Pérez, Ana. (2001). El desarrollo de colección: evaluación en las Bibliotecas españolas. Granada: Universidad de Granada, 2001.

Reglamento General del Sistema Bibliotecario de la Universidad Nacional Autónoma de México. http://www.dgb.u nam.mx/index.php/reglamento-sbunam/reglamento-cap7 (2014-01-16)

Reitz, J.M. (2004). Dictionary for library and information science. USA: Greenwood Publishing Group, 2004.

San Jose, Blanca. (2011). La gestión de la colección cooperativa en las bibliotecas universitarias a comienzos del siglo XXI. Madrid: Departamento de Biblioteconomía y Documentación, 2011.

\section{Apéndice}

Entrevista para los encargados del área de adquisiciones de las bibliotecas

El presente cuestionario tiene como objetivo recabar información sobre el desempeño de los proveedores en la venta de libros a las bibliotecas especializadas del área de humanidades de la UNAM.

1. ¿La biblioteca ha invitado a un proveedor a que se registre en la UNAM para la venta de libros?

2. ¿Cómo se da a conocer a los proveedores las líneas de investigación o necesidades de información de la comunidad académica?

3. ¿Qué porcentaje del material avistas y catálogos que presentan los proveedores para la compra de libros se vincula a las líneas de Investigación del Instituto o Centro?

4. ¿Cree que los proveedores lo alertan de las novedades que están por publicarse?

5. ¿Considera que los proveedores están al día de las novedades que se publican?

6. ¿Estima que la lista de proveedores cubre el área geográfica de interés para la comunidad académica del Instituto o Centro?

7. ¿Con que frecuencia compra libros a proveedores no autorizados por la DGB?

Nombre de la biblioteca:

Entrevista para los proveedores autorizados por la DGB de la UNAM para la compra de libros

1. ¿La UNAM lo invitó (influencio) a darse de alta como proveedor?

2. ¿Conoce las líneas de investigación o temas de interés de las bibliotecas del área humanística de la UNAM?

3. ¿Qué perfil tiene el personal que selecciona el material a presentar en las bibliotecas especializadas del área humanística de la UNAM?

4. ¿Cómo eligen las editoriales que ofrecen a la UNAM?

5. Las editoriales que maneja, ¿son especializadas en las líneas de investigación de las bibliotecas que atiende?

6. ¿Qué proceso aplican para detectar las novedades bibliográficas?

7. ¿Qué área geográfica abarcan las editoriales que maneja?

8. ¿A qué bibliotecas del área humanística asiste más en la venta de libros?

Nombre del proveedor:

Enviado: 2014-04-01. Segunda versión: 2014-07-05. Aceptado: 2014-07-11. 